\title{
Visual evoked and emitted potentials and stimulus significance
}

\author{
D. S. RUCHKIN \\ Departments of Phy'siology and Computer Science \\ Lniversity of Marnland, Baltimore, Md. 21201 \\ and \\ S. SUTTON \\ Biometrics Research Unit \\ New York State Department of Mental Hygiene \\ New York, N.Y. 10032
}

Sutton et al (1967) found that an emitted potential would occur in the absence of an auditory stimulus when the absence provided significant information. This result is extended to visual stimuli and is shown to be statistically highly significant. The contribution of eye-movement artifact to the results is shown to be negligible.

There have been several reports of changes in human cerebral potentials occurring at about the time of an expected but absent stimulus (Rusinov, 1959; Sutton et al, 1967; Klinke et al, 1968; Barlow, 1969; Weinberg et al, 1970: Picton et al, 1973). Such events are clear signs of endogenous processes, apparently dependent to a large degree upon memory and internal time estimation mechanisms. Following the terminology of Weinberg et al, we will refer to such activity as emitted potentials, in contrast to potentials which are evoked by a stimulus.

Sutton et al (1967) found that an emitted potential would appear in the absence of a click when that absence provided significant information. In their paradigm, the $\mathrm{S}$ was instructed to guess, before each trial, whether the stimulus would consist of a single click or two clicks, either of which could be loud or soft. The resulting average evoked potentials (AEPs) had a large positive wave at about $300 \mathrm{msec}$ latency (P3) in the epoch following the time of the second click, whether or not the second click had actually been presented. However, when the $\mathrm{S}$ was instructed to guess whether the click intensities would be loud or soft, no P3 appeared in the epoch following the second click, regardless of the presence or absence of the second click. The evoked and emitted P3 components were interpreted as reflections of processes concerned with the information content or significance of the stimulus for the S. In this report, we extend Sutton et al's finding to visual stimuli, demonstrating independence of stimulus modality for emitted P3 components.

\section{METHOD}

Six male students and one female technician with a median age of 23 years (range 21 to 30 ) were paid to serve as Ss.

The Ss were comfortably seated in a quiet, dimly lit room. The stimuli were $10-\mathrm{msec}$ light flashes obtained from a Grass PS-2 photostimulator with an intensity setting of 16 . The lamp was enclosed in a sound-absorbent box. Continuous background noise delivered via a loudspeaker masked residual sounds from the lamp and stimulator. Control recordings indicated that there were no auditory evoked potentials. The lamp face was covered with a sheet of exposed X-ray film, which served to attenuate light intensity. and a mask, which limited the area of illumination to a rectangular patch of $3.5 \times 5.0 \mathrm{~cm}$. The lamp was placed $110 \mathrm{~cm}$ from the $\mathrm{S}$. In order to reduce eye-movement artifacts, the Ss were instructed to fixate upon a 9-mm-square patch in the center of the illumination rectangle.

Trials of one and two flashes were presented in a random sequence generated by a computer pseudorandom number program. One- and two-flash trials were equiprobable. The interval between the two flashes was fixed for each experiment. In different experiments, intervals of 870.880 .890 , and 900 msec were used. Trials were presented in blocks of 50 with an average intertrial interval of $15 \mathrm{sec}$. In the control blocks, the $S$ was informed, prior to each trial, whether there would be one flash or two flashes (certain condition). In the experimental blocks, the $S$ guessed, prior to each trial, whether there would be one flash or two flashes (uncertain condition). A correct guess was rewarded with an addition of $\$ .15$ to the S's payment. An incorrect guess was penalized by a deduction of $\$ .15$. Four certain and four uncertain blocks were presented in counterbalanced order, providing approximately 100 trials of each of the four combinations of certain or uncertain condition and one or two flashes.

The electroencephalogram was recorded from scalp locations at vertex $\left(\dot{C}_{z}\right.$ according to the International 10-20 System) and occipital $\left(\mathrm{O}_{2}\right)$ regions, both referred to the left earlobe $\left(A_{1}\right)$. The forehead was grounded. The vertical EOG was recorded from electrodes above and below the left eye for five Ss. Grass silver disk electrodes were used on the scalp. Beckman miniature biopotential electrodes were used elsewhere. The amplifiers were set to a bandpass of $.1-100 \mathrm{~Hz}$. The data were recorded on analog magnetic tape. AEPs were computed for each of the four conditions: one flash certain, two flashes certain, one flash uncertain, two flashes uncertain. AEPs for the $C_{z}$ electrode were computed on-line. AEPs for the $\mathrm{O}_{z}$ and EOG electrodes were computed from tape playbacks.

\section{RESULTS}

Figure 1 illustrates the AEPs recorded from one S. For both $\mathrm{C}_{\mathrm{z}}$ and $\mathrm{O}_{\mathrm{z}}$, the AEPs for the four conditions are markedly different in the epoch following the time at which the second flash occurs. In the certain condition, a response appears after the second flash while no response is discernible in the corresponding epoch of the one-flash average. In the uncertain condition, the second flash evokes a large $\mathrm{P} 3$ component with a peak latency of $360 \mathrm{msec}$ at both $\mathrm{C}_{\mathrm{z}}$ and $\mathrm{O}_{z}$. An emitted P3 appears in the uncertain condition one-flash AEP, with a peak latency of $400 \mathrm{msec}$ at $\mathrm{C}_{z}$ and $480 \mathrm{msec}$ at $\mathrm{O}_{z}$ following the time of the omitted second flash. Its magnitude is smaller and its shape is less sharply peaked than the average evoked P3 in the two-flash condition. The contribution of eye-movement artifact to the scalp recordings is negligible. ${ }^{1}$ Similar results were obtained from five of the other six Ss. The AEPs for the $\mathrm{C}_{z}$ and $\mathrm{O}_{z}$ recordings from the sixth $\mathrm{S}$ (R.R.) were obscured by alpha activity. The experiments were replicated for three Ss. 


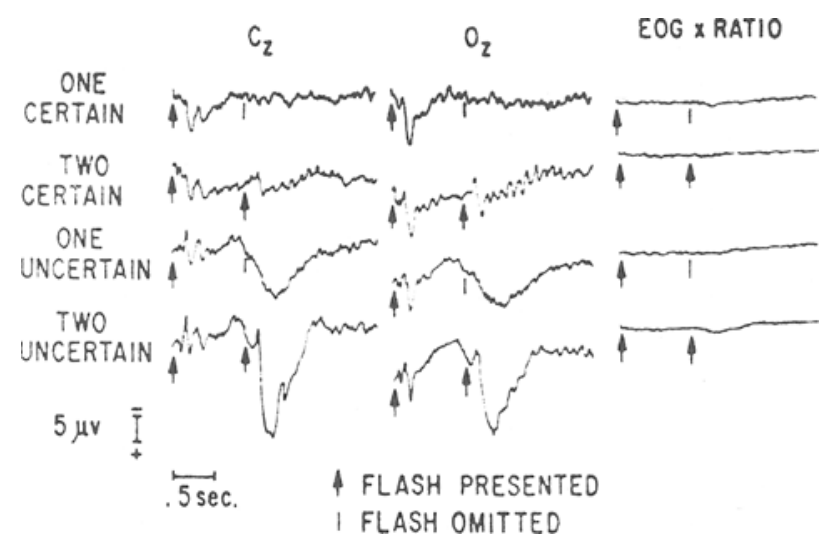

Fig. 1. AEPs recorded from S G.E. Analysis time is 2.5 sec. Each AEP composed of 80 responses. EOG $x$ ratio ${ }^{1}$ indicates the probable effective contribution of vertical EOG to $C_{z}$ recording. Contribution to $\mathrm{O}_{\mathrm{z}}$ recording is less.

For each $\mathrm{S}$, for the one-flash data recorded from $\mathrm{C}_{2}$, P3 amplitude was measured from baseline at the onset of the first stimulus to the peak positive amplitude between 300 and $450 \mathrm{msec}$ following the time of the second stimulus. The differences between the uncertain condition and the certain condition are presented in Table 1. A t test for paired observations was used to test whether the uncertain condition P3 was more positive than the certain condition $\mathrm{P} 3$. The greater positivity for the uncertain condition was found to be significant at the $p<.0005$ level (one-tailed test).

The contribution of eye-movement artifact to the scalp recording average responses was assessed for five Ss for whom such data were available. Table 1 presents, for the single-flash data, the differences between the average EOG amplitudes in the uncertain condition and the average EOG amplitudes in the certain condition at the same latencies used for the $C_{z}$ computations. A t test for paired observations indicated that the EOG differences were not significantly different from zero. For each S,

Table 1

Differences Between Amplitudes in Microvolts for Single Stimulus, Uncertain Minus Certain

\begin{tabular}{lcccc}
\hline \multicolumn{1}{c}{ S } & $\mathrm{C}_{\mathrm{z}}$ & EOG & $\begin{array}{c}\text { EOG X } \\
\text { Ratio }\end{array}$ & $\begin{array}{c}\text { Latency } \\
(\mathrm{msec})^{*}\end{array}$ \\
\hline M.B. & 9.1 & & & 380 \\
C.R. & 11.2 & & & 430 \\
G.E. & 11.5 & .0 & .0 & 400 \\
C.H. & 6.3 & 5.2 & 1.07 & 320 \\
R.R. & 1.9 & 2.2 & .37 & 340 \\
R.S. & 10.3 & -.9 & -.15 & 350 \\
K.M. & 10.2 & -11.4 & -1.95 & 380 \\
AV & 8.6 & -1.0 & -.15 & 371 \\
SD & 3.4 & 6.3 & & \\
t & 6.64 & -.356 & & \\
df & 6 & 4 & & \\
p (one-tail) & .0005 & n.s. & & \\
\hline
\end{tabular}

Note-AEPs and EOGs are measured at peak latency of emitted $P 3$ in the uncertain condition.

*Latency from time of omitted tlash. the EOG difference was adjusted by its probable contribution to the $\mathrm{C}_{\mathrm{z}}$ ratio. ${ }^{1}$ The resulting values, the probable effective contribution of the vertical EOG to the $\mathrm{C}_{\mathrm{z}}$ recording, are presented in Table 1 . It can be seen that eye-movement artifact at most would make a minor contribution to the $\mathrm{C}_{\mathbf{z}}$ potential difference.

The form and latency of the average emitted P3s recorded from $\mathrm{O}_{\mathrm{z}}$ were similar to the corresponding emitted P3s of the $\mathrm{C}_{z}$ AEPs. However, the $\mathrm{O}_{z}$-emitted potential amplitudes were lower than the $\mathrm{C}_{\mathrm{z}}$-emitted potential amplitudes, the ratio of $\mathrm{O}_{z}$ to $\mathrm{C}_{\mathrm{z}}$ ranging from .56 to .90 . This ratio is consistent with Vaughan's (1969) isopotential map for the P3 component.

\section{DISCUSSION}

It has been demonstrated that the emitted potential phenomenon for auditory stimuli also occurs in response to visual stimuli. The emitted P3 corresponding to the time of the second stimulus is of lower amplitude and broader duration than the P3 evoked by the second flash. Four of the Ss in this study participated in parallel click-emitted potential experiments. Figure 2 provides a comparison for one $S$ of click- and flash-emitted potentials recorded from $C_{z}$. By inspection, they appear quite similar, although a systematic study would be required to decide if they are, in fact, identical.

Vaughan (1969) and Vaughan \& Ritter (1970) presented isopotential plots of evoked P3 components for both visual and auditory stimuli. They suggest a parieto-temporal cortex source for P3. (It was pointed out that the isopotential plots were also compatible with a deep, subcortical source, but it was reasoned that it was unlikely for the source to be subcortical.) The relative amplitudes of the $\mathrm{C}_{z}$ and $\mathrm{O}_{z}$ flash-emitted $\mathrm{P} 3$ components are compatible with Vaughan and Ritter's isopotential plots and consequently suggest that the source of these emitted potentials may be in the parieto-temporal cortex.

Sutton et al (1967) conjectured that the evoked and emitted P3s reflected the same process. The lower amplitude and broader duration obtained for the average emitted P3 than for the average evoked $\mathrm{P} 3$ was attributed to the lack of a precisely timed external stimulus. Instead, the $S$ had to utilize internal time estimation to decide that the trial consisted of a single stimulus. Variation in such time estimation could lead to asynchrony in
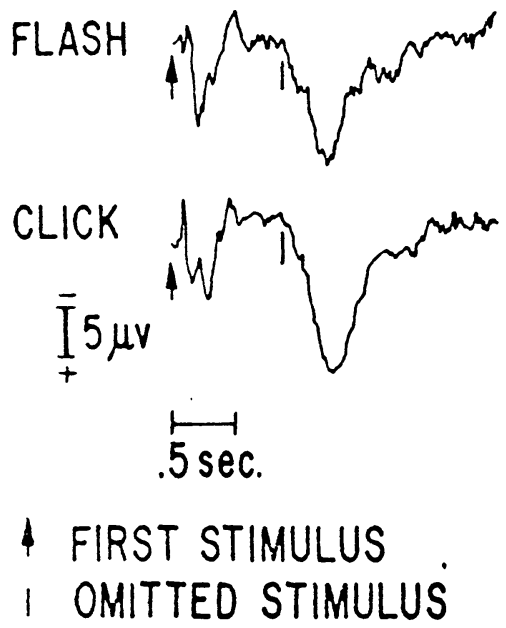

Fig. 2. AEPs for the one-stimulus, uncertain condition recorded from $C_{z}$ of $S$ R.S. Analysis time is 2.5 sec. Each AEP composed of 95 responses. 
the emitted P: component and consequently a lower amplitude. broader duration component average. Picton et al (1973) have presented data that support this interpretation. However. the question still remains as to whether the individual emitted P3s are of the same shape as the individual evoked P3s and that the averages differ due to latency variation of the $\mathrm{P} 3$ component. or whether the individual emitted P3s are each of lower amplitude and broader duration. perhaps due to asynchrony at the unit level.

Paul \& Sutton (1972) interpreted the P3 activity as a reflection of the information content or salience of the stimulus for the S. Karlin (1970) and Donchin \& Smith (1970) have argued that P3 may be determined by processes such as the CNV which precede the stimulus. This view is not supported by the experiments of Donald \& Goff (1971) and Tueting \& Sutton (1973). who have shown that orderly relations can be found between the amplitude of P3 and experimental variables. independently of the level of the CNV. However, the question remains open whether the $\mathrm{P} 3$ response to an absent stimulus may be a special case and. in fact. represents the return of the CNV to baseline. The CNV duration might in this context be viewed as a good candidate for a cortical correlate of the S's time estimation process. Further experimentation is needed to resolve this issue.

\section{REFERENCES}

Barlow, J. S. Some observations on the electrophysiology of timing in the central nervous system. Electroencephalography \& Clinical Neurophysiology, $1969,27,545$.

Corby, J. C., \& Kopell, B.S. Differential contributions of blinks and vertical eye movements as artifacts in EEG recording. Psychophysiology, 1972, 6, 640-644.

Donald, M. W., \& Goff, W. R. Attention related increases in cortical responsivity dissociated from the contingent negative variation. Science, 1971, 172, 1163-1166.

Donchin, E., \& Smith. D. B. D. The contingent negative variation and the late positive wave of the average evoked potential. Electroencephalography \& Clinical Neurophysiology, 1970 , 29, 201-203.

Girton, D. G., \& Kamiya. J. A simple on-line technique for removing eye movement artifacts from the EEG. Electroencephalography \& Clinical Neurophysiology, 1973, 34, 212-216.

Karlin, L. Cognition, preparation and sensory evoked potentials. Psychological Bulletin, 1970, 73, 122-136.

Klinke, R., Fruhstorfer, H., \& Finkenzeller, P. Evoked responses as a function of external and stored information. Electroencephalography \& Clinical Neurophysiology. 1968. $25,119-122$.

Paul, D. D., \& Sutton, S. Evoked potential correlates of response criterion in auditory signal detection. Science, 1972, 177 , 362-364.

Picton, T. W., Hillyard, S. A., \& Galambos, R. Cortical evoked responses to omitted stimuli. In $M$. N. Livanov (Ed.), Hajor problems of brain electrophysiology. Moscow: USSR Academy of Sciences, 1973 , in press.

Rusinov, V. S. Electroencephalographic studies in conditioned reflex formation in man. In $\mathrm{M}$. A. B. Brazier (Ed.), The central nerious system and behavior. (Trans.) Madison, N.J: Second Macy Conference, 1959. Pp. 249-256.

Sutton, S., Tueting. P., Zubin, J., \& John, E. R. Evoked potential correlates of stimulus uncertainty. Science, 1967. 155, 1436-1439.

Tueting, P., \& Sutton, S. The relationship between pre-stimulus negative shifts and post-stimulus components of the averaged evoked potential. In S. Kornblum (Ed.), Attention and performance IV. New Y ork: Academic Press, 1973.

Vaughan, Jr., H. G. The relationship of brain activity to scalp recordings of event-related potentials. In $E$. Donchin and $D . B$. Lindsley (Eds.), A verage evoked potentials. Washington D.C: Government Printing Office, 1969.

Vaughan, Jr., H. G., \& Ritter, W. The sources of auditory evoked responses recorded from the human scalp. Electroencephalography \& Clinical Neurophysiology, 1970, 28, 360-367.

Weinberg, H., Walter, W. G., \& Crow, H. J. Intracerebral events in humans related to real and imaginary stimuli. Electroencephalography \& Clinical Neurophysiology, 1970, 29, 1-9.

\section{NOTE}

1. Corby \& Kopell (1972) and Girton \& Kamiya (1973) have demonstrated that the amplitude of eye-movement artifact recorded at the scalp is a fixed ratio of the EOG amplitude. Consequently, such ratios were determined for $\mathrm{C}_{z}$ and $\mathrm{O}_{z}$ for the five $S$ s from whom the EOG was recorded. The ratio range was .15 to .21 for $C_{z}$ and .08 to .125 for $O_{z}$. The probable effective contribution of EOG to the average $C_{z}$ amplitude was computed by multiplying the average EOG amplitude by the previously determined ratio of $C_{7}$ to EOG eye-movement potentials. The $C_{z}$ and $\mathrm{O}_{z}$ ratios were .178 and .122 , respectively, for G.E., whose data is illustrated in Fig. 1. The ratio of .178 was used for the EOG $x$ ratio waveforms in Fig. 1 .

(Received for publication May 16, 1973.)

\section{Children's discrimination learning as related to delayed punishment*}

\section{KENNETH L. WITTE $†$ and ROBERT K. JOHNSON \\ University of Arkansas, Fayetteville, Ark. 72701}

The performances of 80 third-grade children on either an easy or a difficult discrimination task were compared under two delay of punishment conditions, 0 or $20 \mathrm{sec}$. Punishment consisted of a $3-\mathrm{sec} 98-\mathrm{dB}$ tone. Immediate punishment resulted in better discrimination performance, i.e., fewer trials to criterion and more correct responses, than did delayed punishment. The magnitude of the delayed punishment effect was

* Joel S. Freund sponsors this paper and takes full editoria responsibility for it. This study was the junior author's thesis submitted in partial fulfillment of the requirements for the degree of Master of Arts under the direction of the senior author.

tRequests of reprints should be sent to $K$. L. Witte Psychology Department, University of Arkansas, Fayetteville, Ark. 72701. independent of the difficulty of the discrimination task. On the bases of additional data, it was suggested that the effects of delayed punishment upon children's discrimination learning involve an attentional rather than a motivational mechanism.

Discrimination learning research with young children has demonstrated that delayed reward for correct responses has a deleterious effect (see Reese \& Lipsitt, 1970 , pp. 188-189), but at the present time there is no conclusive evidence that delayed punishment for errors produces a learning decrement. Apparently, the effect of delayed punishment upon children's discrimination learning has been examined only twice (Walters, 1964: Setterington \& Walters, 1964). Factorial designs employing concurrent delayed reward and delayed punishment were used in these two studies; the delay intervals were 0,10 , and $30 \mathrm{sec}$ and 0,10 , and $20 \mathrm{sec}$, 